\title{
ROTATION METHOD FOR RECONSTRUCTING PROCESS AND FIELD FROM IMPERFECT DATA
}

\author{
PETER C. CHU and LEONID M. IVANOV \\ Naval Ocean Analysis and Prediction Laboratory, \\ Department of Oceanography, Naval Postgraduate School, \\ Monterey, California, USA \\ TATYANA M. MARGOLINA \\ Marine Hydrophysical Institute of the Ukrainian Academy of Sciences, \\ Sevastopol, Crimea, Ukraine
}

Received February 12, 2003; Revised August 28, 2003

\begin{abstract}
Reconstruction of processes and fields from noisy data is to solve a set of linear algebraic equations. Three factors affect the accuracy of reconstruction: (a) a large condition number of the coefficient matrix, (b) high noise-to-signal ratio in the source term, and (c) no a priori knowledge of noise statistics. To improve reconstruction accuracy, the set of linear algebraic equations is transformed into a new set with minimum condition number and noise-to-signal ratio using the rotation matrix. The procedure does not require any knowledge of low-order statistics of noises. Several examples including highly distorted Lorenz attractor illustrate the benefit of using this procedure.
\end{abstract}

Keywords: Reconstruction; imperfect data; linear/nonlinear models.

\section{Introduction}

Reconstruction (reproduction from noisy data) of processes and fields in modern physics, geophysics, astrophysics, plasma physics and other disciplinary sciences is to solve an ill-posed linear algebraic equation,

$$
\mathbf{A} \hat{\mathbf{a}}=\mathbf{Q} \mathbf{Y},
$$

where $\hat{\mathbf{a}}$ is the estimated state vector ( $L$ dimensional) for the exact state vector $\mathbf{a} ; \mathbf{A}$ is a $P \times L$ coefficient matrix, $\mathbf{Q}$ is a $P \times P$ square matrix $(P>L) ; \mathrm{Y}$ is a $P$-dimensional observation vector, consisting of a signal $\overline{\mathbf{Y}}$ and a noise $\mathbf{Y}^{\prime}$,

$$
\mathbf{Y}=\overline{\mathbf{Y}}+\mathbf{Y}^{\prime} \text {. }
$$

The two known matrices $\mathbf{A}$ and $\mathbf{Q}$ are determined by the physical process or field. Let $\|\ldots\|$ be the
Euclidean norm and

$$
\begin{gathered}
\eta_{1}=\frac{\left\|\mathbf{Q} \mathbf{Y}^{\prime}\right\|}{\|\mathbf{Q} \overline{\mathbf{Y}}\|}, \quad \eta_{2}=\frac{L}{P}, \\
\eta_{3}=\frac{\max (\text { singular values of } \mathbf{A})}{\min (\text { singular values of } \mathbf{A})},
\end{gathered}
$$

be the noise-to-signal ratio, dimension ratio and condition number of the matrix A. For a particular system, $\eta_{2}$ is given.

Usually, $\eta_{1}$ and $\eta_{3}$ are large (called "imperfect"),

$$
\eta_{1} \geq 1, \quad \eta_{3} \gg 1,
$$

which makes (1) difficult to solve. Besides, the loworder noise statistics and the norm of the exact state vector $(\|\mathbf{a}\|)$ are unknown. Reduction of $\eta_{1}$ and $\eta_{3}$ (i.e. reduction of imperfection) without a priori knowledge of noise statistics and $\|\mathbf{a}\|$ is an important step toward solving (1) accurately. If the 
matrix $\mathbf{A}$ has no noise, the accuracy in determining $\hat{\mathbf{a}}$ is given by [Tikhonov et al., 1990],

$$
\|\gamma\|^{2}=\frac{\|\mathbf{a}-\hat{\mathbf{a}}\|^{2}}{\|\mathbf{a}\|^{2}} \leq \eta_{1}^{2} \eta_{3}^{2} .
$$

Traditional regularization methods (e.g. [Tikhonov \& Arsenin, 1979; Bennett, 1992]) transform (1) into a system with stable solution to small perturbations in $\mathbf{Q}$ and $\mathbf{Y}$. However, they lead to biased estimations â for $\mathbf{a}$ and do not guarantee

$$
\hat{\mathbf{a}} \rightarrow \mathbf{a} \text { as } \eta_{1} \rightarrow 0 \text {. }
$$

Furthermore, the traditional regularization methods require a priori knowledge of $\|\mathbf{a}\|$.

To overcome these weaknesses, a new rotation method for $\eta_{2}<1$ is developed in this study to change (1) into a new system with possibly minimum coefficient matrix and noise-to-signal ratio without a priori knowledge of noise statistics.

\section{Rotation Method}

When the dimension ratio $\eta_{2}(<1.0)$ is given, large values of $\eta_{1}$ and $\eta_{3}$ (called "imperfect" data),

$$
\eta_{1} \geq 1, \quad \eta_{3} \gg 1
$$

make (1) difficult to solve. Note that the low-order noise statistics and the norm of the exact solution $(\|\mathbf{a}\|)$ are usually unknown. Reduction of $\eta_{1}$ and $\eta_{3}$ (i.e. reduction of imperfection) without knowing noise statistics and $\|\mathbf{a}\|$ is an important step toward solving (1).

Nonsingular orthogonal transformation is conducted through multiplication of (1) by a plane rotation matrix $\mathbf{S}$ from the left,

$$
\text { SAâ }=\mathbf{S Q Y} \text {, }
$$

which changes the coefficient matrix and the source term from (A, $\mathbf{Q Y})$ to (SA, $\mathbf{S Q Y})$ and provides the opportunity to minimize the imperfection of the new system (3),

$$
\tilde{\eta}_{3}^{2}\left(1+\tilde{\eta}_{1}^{2}\right) \rightarrow \min ,
$$

where

$$
\tilde{\eta}_{1} \equiv \frac{\left\|\mathbf{S Q} \mathbf{Y}^{\prime}\right\|}{\|\mathbf{S Q} \overline{\mathbf{Y}}\|}, \quad \tilde{\eta}_{3} \equiv \frac{\|\mathbf{S Q} \overline{\mathbf{Y}}\|}{\|\mathbf{a}\|} .
$$

Minimization (4) is obtained by the following maximization [Strakov, 1991; Ivanov et al., 2001]

$$
J_{1}=\|\mathbf{A}\|^{2}-\frac{\|\mathbf{S Q Y}\|^{2}}{\|\mathbf{a}\|^{2}} \rightarrow \max ,
$$

where $\|\mathbf{A}\|$ is the spherical norm of the matrix $\mathbf{A}$. Substitution of (5) into (6) leads to

$$
J_{1}=\|\mathbf{A}\|^{2}-\tilde{\eta}_{3}^{2}\left[1+\frac{2\left(\mathbf{S Q} \overline{\mathbf{Y}}^{*} \mathbf{S Q Y} \mathbf{Y}^{\prime}\right)}{\|\mathbf{S Q} \overline{\mathbf{Y}}\|^{2}}+\tilde{\eta}_{1}^{2}\right] \rightarrow \max
$$

which is the procedure to obtain minimum values of $\tilde{\eta}_{1}$ and $\tilde{\eta}_{3}$ without knowing $\|\mathbf{a}\|^{2}$. Here, the symbol "*" indicates the scalar product in the Euclidean space. For a white noise $\mathbf{Y}^{\prime}$, we have

$$
\left(\mathbf{S Q} \overline{\mathbf{Y}}^{*} \mathbf{S Q Y}^{\prime}\right) \rightarrow 0 \text { as } P \rightarrow \infty \text {. }
$$

The maximization of $J_{1}$ using ( 7 ) is then equivalent to the minimization (4). Note that this minimization is not necessarily the same as

$$
\tilde{\eta}_{3}^{2} \rightarrow \min , \quad \tilde{\eta}_{1}^{2} \rightarrow \min .
$$

\section{Accuracy}

The accuracy of the reconstruction (1) is estimated by (2) with the given norm of the exact state vector $\|\mathbf{a}\|$. Since $\|\mathbf{a}\|$ is not a priori known, an effective norm $\left\|\mathbf{a}_{\text {eff }}\right\|$ is defined by the following minimization process,

$$
J_{2}=\left|\frac{\|\hat{\mathbf{a}}\|^{2}}{\left\|\mathbf{a}_{\mathrm{eff}}\right\|^{2}}-1\right| \rightarrow \min ,
$$

Simple analysis on (4)-(8) shows that two norms $\left\|\mathbf{a}_{\text {eff }}^{*}\right\|$ and $\left\|\mathbf{a}_{\text {eff }}^{* *}\right\|$ (generally $\left.\left\|\mathbf{a}_{\text {eff }}^{*}\right\| \neq\left\|\mathbf{a}_{\text {eff }}^{* *}\right\|\right)$ exist that the functionals $J_{1}$ and $J_{2}$ reach their maximum values, respectively, and

$$
\|\mathbf{a}\| \leq\left\|\mathrm{a}_{\text {eff }}^{*}\right\|, \quad\left\|\mathrm{a}_{\text {eff }}^{* *}\right\| \leq 2\|\mathbf{a}\| .
$$

If $\|\mathbf{a}\| \rightarrow\left\|\mathbf{a}_{\text {eff }}^{*}\right\|$ then $\|\gamma\|^{2} \rightarrow 0$. When $\|\mathbf{a}\| \rightarrow\left\|\mathbf{a}_{\text {eff }}^{* *}\right\|$ we have

$$
\|\gamma\|^{2} \rightarrow\left[1-\frac{\left\|\mathrm{a}_{\mathrm{eff}}^{* *}\right\|}{\|\mathrm{a}\|}\right]^{2}
$$

Therefore, replacement of $\|\mathbf{a}\|$ by $\left\|a_{\text {eff }}^{* *}\right\|$ will not deteriorate the accuracy of the solution of (1). A similar replacement of $\|\mathbf{a}\|$ is also suggested for colored noises and deterministic perturbations. However, the reconstruction accuracy might deteriorate because the scalar product $\left(\mathbf{S Q} \overline{\mathbf{Y}}^{*} \mathbf{S Q} \mathbf{Q} \mathbf{Y}^{\prime}\right)$ does not tend to 0 for $P \rightarrow \infty$.

\section{Applications}

The new transformed system (3) can be solved by usual algebraic methods such as the Gauss method. For simplicity, we restrict the reconstruction to data distorted by the white noise only. 


\subsection{Example 1: Linear scalar process}

Consider a temporally varying linear scalar process,

$$
\bar{Y}(t)=\alpha+\beta t, \quad t \in[0, T]
$$

perturbed by a white noise $Y^{\prime}(t)$. The noisy data are represented by

$$
Y(t)=\bar{Y}(t)+Y^{\prime}(t)
$$

The Fourier series

$$
X(t)=a_{0}+\sum_{i=1}^{38}\left[a_{i} \sin \left(\frac{i \pi t}{T}\right)+a_{i+19} \cos \left(\frac{i \pi t}{T}\right)\right],
$$

is used to recover the signal $\bar{Y}(t)$. The reconstruction is to determine the estimated state vector

$$
\hat{\mathbf{a}}=\left(a_{1}, a_{2}, \ldots, a_{L}\right), \quad L=38,
$$

such that the temporally integrated difference between $X(t)$ and $Y(t)$ reaches the minimum value,

$$
J=\int_{0}^{T}[X(t)-Y(t)]^{2} d t \rightarrow \min .
$$

Substitution of (9) into (10) leads to Eq. (1) of â with a very high condition number for the coefficient matrix.

The noisy dataset $Y(t)$ is generated in such a way that the sensitivity of reconstruction quality to $\left(\eta_{1}, \eta_{2}\right)$ can be investigated: $60-100$ points (varying $\mathrm{P}$ and time step $\Delta t$ ) uniformly distributed into the interval $[0, T]$ are used to get $\bar{Y}(t)$, and white noises $Y^{\prime}(t)$ with $\eta_{1}$ ranging from 0 to 3 are added to $\bar{Y}(t)$. Here, $T / 60 \geq \Delta t \geq T / 100$.

The three nondimensional parameters $\eta_{1}, \eta_{2}$ and $\eta_{3}$ vary within the following ranges,

$$
0 \leq \eta_{1} \leq 3 ; \quad 0.4 \leq \eta_{2} \leq 0.6 ; \quad \eta_{3} \simeq 10^{6} .
$$

For such a high condition number, Eq. (1) cannot be solved directly. The rotation matrix $\mathbf{S}$ is calculated using the maximization procedure depicted in Sec. 2. The condition number of the coefficient matrix of the new system (3) reduces to

$$
\tilde{\eta}_{3} \simeq 1.5 \text {. }
$$

The reconstructed accuracy $\|\gamma\|$ reduces with the decrease of $\eta_{1}$ (noise-to-signal ratio before the rotation) monotonically from 4.0 for $\eta_{1}=3.0$ to 0 for

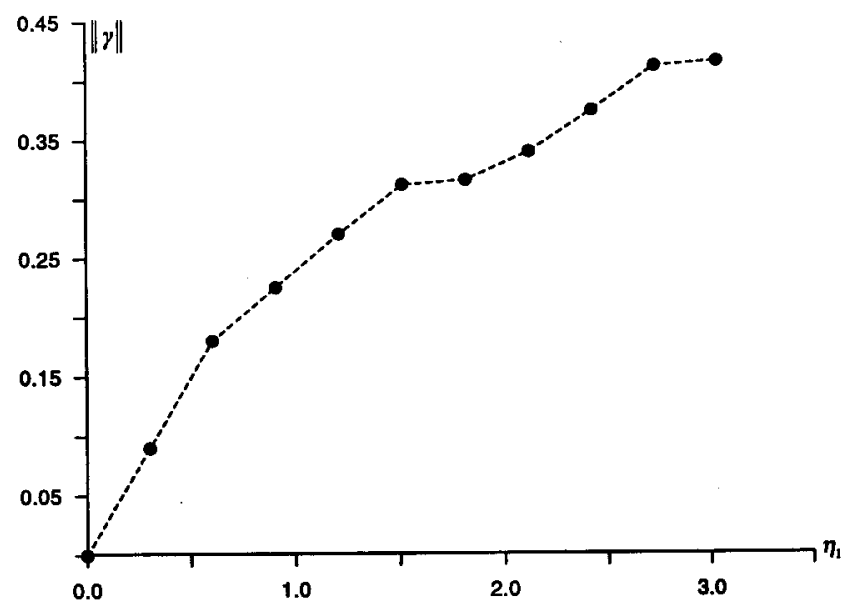

(a)

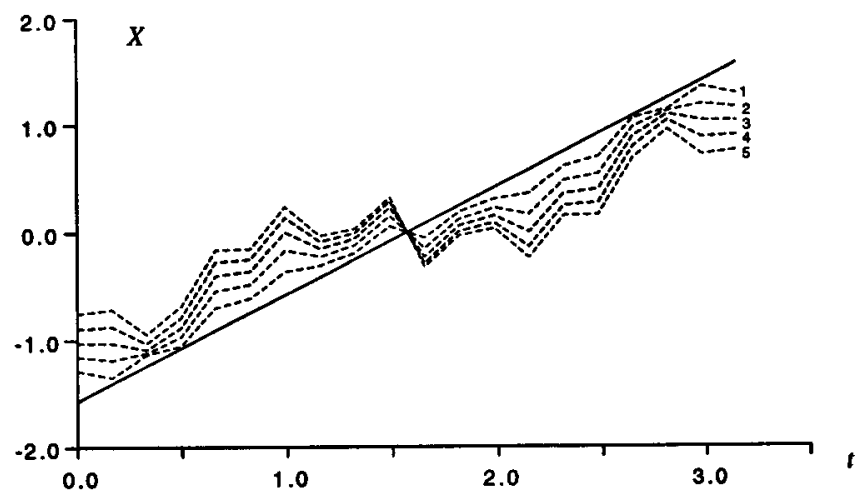

(b)

Fig. 1. Reconstruction of the one-dimensional linear process: (a) upper panel: reconstruction accuracy, and (b) lower panel: comparison between $\bar{Y}(t)$ (solid line) and reconstructed processes $X(t)$ (dashed curves) with 1, 2, 3, 4 and 5 corresponding to $\eta_{1}=0.6,1.2,1.8,2.4$, and 3.0.

$\eta_{1}=0$ [Fig. 1(a)]. The reconstructed process $\hat{X}(t)$ is closer to $\bar{Y}(t)$ as $\eta_{1}$ reduces [Fig. 1(b)].

\subsection{Example 2: Two dimensional field}

Eremeev et al. [1992] reconstructed the Black Sea summer climatological surface temperature field $\left(T_{\text {clim }}\right)$ using the traditional regularization method [Bennett, 1992]. The nondimensional parameters $\left(\eta_{1}, \eta_{2}, \eta_{3}\right)$ vary within the following intervals:

$$
\begin{gathered}
0 \leq \eta_{1} \leq 4, \quad 0.025 \leq \eta_{2} \leq 0.67, \\
3 \times 10^{4} \leq \eta_{3} \leq 3 \times 10^{7} .
\end{gathered}
$$

For large values of $\left(\eta_{1}, \eta_{3}\right)$, the accuracy of the traditional regularization methods is not very good.

Consider that the climatological surface temperature field $T_{c l i m}(x, y)$ is perturbed by white 


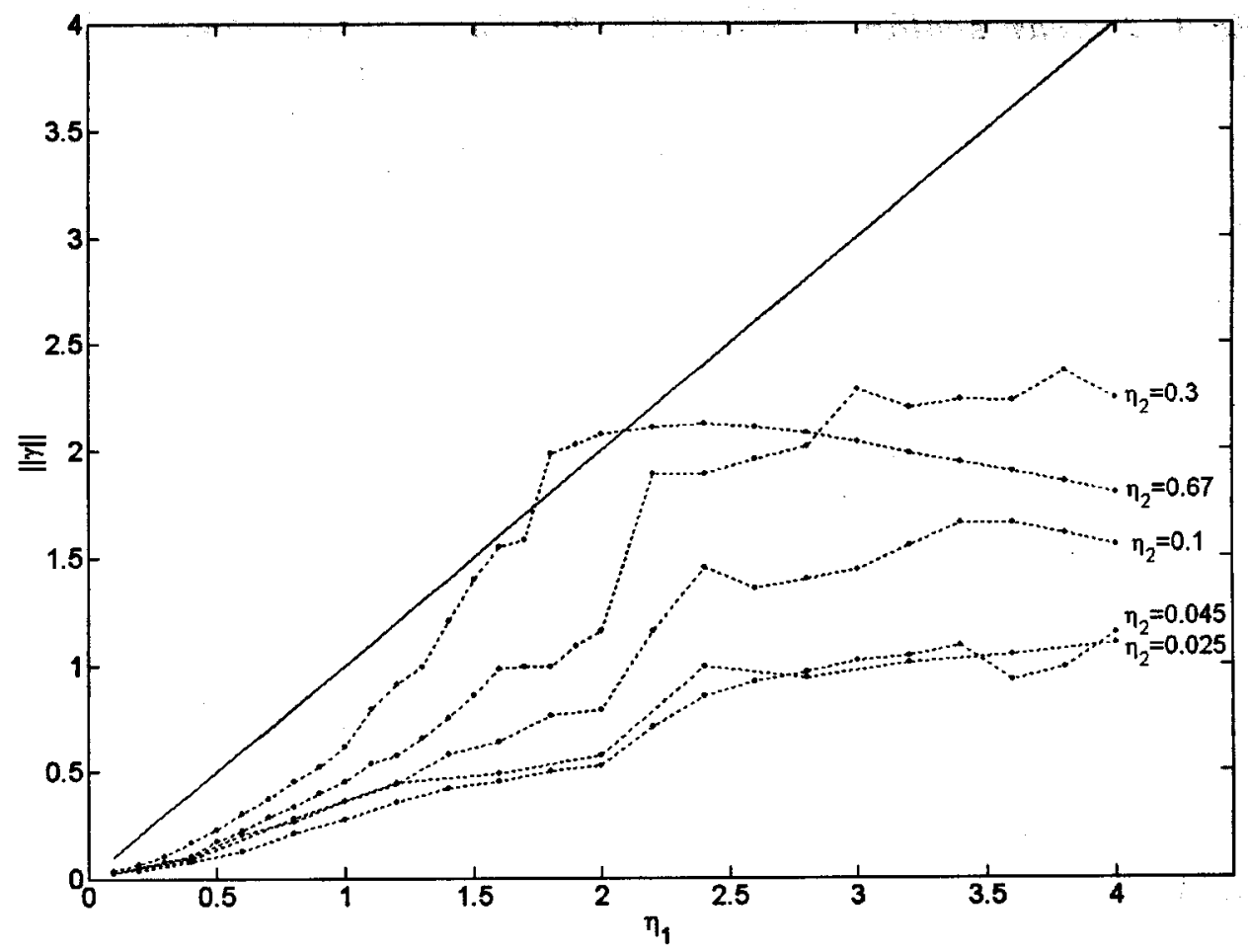

Fig. 2. Dependence of the accuracy $\|\gamma\|$ on $\eta_{1}$ with different $\eta_{2}$-values for the two-dimensional temperature field in the Black Sea. Note that $\|\gamma\|$ is usually smaller than $\eta_{1}$.

noises $T^{\prime}(x, y)$. The noisy data are represented by $T_{\text {data }}(x, y)=T_{\text {clim }}(x, y)+T^{\prime}(x, y), \quad(x, y) \in \Omega$, where $\Omega$ is the Black Sea. The generalized Fourier series,

$$
T(x, y)=\sum_{i=1}^{30} a_{i} \psi_{i}(x, y),
$$

is used to recover the signal $T_{c \lim }(x, y)$. Here, the basis functions $\left\{\psi_{i}(x, y), i=1,2, \ldots, 30\right\}$ are the eigenfuctions of the plane von Neumann operator with homogeneous boundary conditions for the domain $\Omega$ [Eremeev et al., 1992]. The reconstruction is to determine the estimated state vector

$$
\hat{\mathbf{a}}=\left(a_{1}, a_{2}, \ldots, a_{L}\right), \quad L=30,
$$

such that the spatially integrated difference between $T_{\text {data }}(x, y)$ and $T(x, y)$ reaches the minimum value, $J=\iint_{\Omega}\left[T(x, y)-T_{\mathrm{data}}(x, y)\right]^{2} d x d y \rightarrow \min$

Substitution of (13) into (14) leads to Eq. (1) of â with a very high condition number for the coeffcient matrix [see (12)].

After applying the rotation matrix $\mathbf{S}$ with the maximization procedure [Eq. (7)], the condition number of the coefficient matrix of the new system (3) reduces to

$$
\tilde{\eta}_{3} \leq 5.0 \text {. }
$$

The reconstructed accuracy $\|\gamma\|$ of our reconstruction scheme is up bounded by $\eta_{1}$ (noise-tosignal ratio before the rotation)

$$
\|\gamma\| \leq \eta_{1}
$$

and usually decreases with decreasing $\eta_{1}$ and $\eta_{2}$ (Fig. 2). Note that the approach does not improve the reconstruction accuracy for $\eta_{2} \leq 0.045$. For large noise-to-signal ratio $\left(\eta_{1}>3.0\right)$, the reconstruction accuracy improves when the dimension ratio $\eta_{2}$ increases from 0.3 to 0.67 .

\subsection{Example 3: Lorenz Attractor}

Lorenz system, a truncated three-component atmospheric convection model, is represented by a threedimensional vector

$$
\mathbf{X} \equiv\left[\begin{array}{l}
X_{1} \\
X_{2} \\
X_{3}
\end{array}\right]
$$


which satisfies the following nonlinear ordinary differential equation with the initial condition

$$
\frac{d \mathbf{X}}{d t}=\mathbf{F}(\mathbf{X}, \mathbf{a}), \quad \mathbf{X}\left(t_{0}\right)=\mathbf{X}_{\mathbf{0}}
$$

where the vector functional $\mathbf{F}$ and state parameter vector are defined by [Lorenz, 1963]

$$
F=\left[\begin{array}{c}
-a_{1} X_{1}+a_{1} X_{2} \\
-X_{1} X_{2}+a_{2} X_{1}-X_{2} \\
X_{1} X_{2}-a_{3} X_{3}
\end{array}\right], \quad \mathbf{a} \equiv\left[\begin{array}{c}
a_{1} \\
a_{2} \\
a_{3}
\end{array}\right]
$$

The initial condition $\left(\mathbf{X}_{0}\right)$ and the parameter vector (a) affect the characteristics of the Lorenz system. Chu [1999] showed that boundary condition was represented by the model parameter. We integrate Eq. (15) with the time step $\Delta t=0.01$ and

$$
\mathbf{X}_{0}=\left[\begin{array}{l}
0.1 \\
0.1 \\
0.1
\end{array}\right], \quad \mathbf{a} \equiv\left[\begin{array}{c}
10 \\
28 \\
8 \\
3
\end{array}\right]
$$

to obtain the famous butterfly pattern for the track in the phase space $\left(X_{1}, X_{2}\right)$ [Fig. 3(a)] and unstable oscillation for temporal variation of $X_{3}$ [Fig. 3(b)].

The observation vector of the Lorenz system $[\mathbf{Y}(t)]$ contains signal $[\overline{\mathbf{Y}}(t)]$ and white noises $\left[\mathbf{Y}^{\prime}(t)\right]$,

$$
\mathbf{Y}(t)=\overline{\mathbf{Y}}(t)+\mathbf{Y}^{\prime}(t)
$$

with $\eta_{1}=0.6$. The butterfly pattern is totally destroyed in the phase space $\left(Y_{1}, Y_{2}\right)$ [Fig. 4(a)]. The time series of the third component $\left(Y_{3}\right)$ shows a stochastic process [Fig. 4(b)].

The signal $\overline{\mathbf{Y}}(t)$ is recovered from the noisy data $\left[Y_{1}(t), Y_{2}(t), Y_{3}(t)\right]$ (Fig. 4) through accurate estimation of the parameter vector $\hat{a}$ and effective reduction of noises. Estimation of the state vector

$$
\hat{\mathbf{a}}=\left(a_{1}, \ldots, a_{L}\right), \quad L=3,
$$

is conducted using the minimization of the temporally integrated difference between $\mathbf{X}(t)$ and $\mathbf{Y}(t)$,

$$
J=\int_{t_{0}}^{T}[\mathbf{X}(t)-\mathbf{Y}(t)]^{*}[\mathbf{X}(t)-\mathbf{Y}(t)] d t \rightarrow \min
$$

Different from Example 2, there is no explicit relationship between $\mathbf{X}(t)$ and $\mathbf{a}$. The minimization (18) becomes

$$
\frac{\partial J}{\partial \hat{a}_{j}}=0
$$

An iterative algorithm [Eykhoff, 1973] and the initial condition

$$
\mathbf{X}\left(t_{0}\right)=\mathbf{Y}\left(t_{0}\right)
$$

are used. Let the parameter vector be estimated by $\hat{\mathbf{a}}^{(n-1)}$ for the $(n-1)$ th iteration. Integration of the Lorenz system

$$
\frac{d \mathbf{X}}{d t}=\mathbf{F}(\mathbf{X}, \hat{\mathbf{a}})
$$

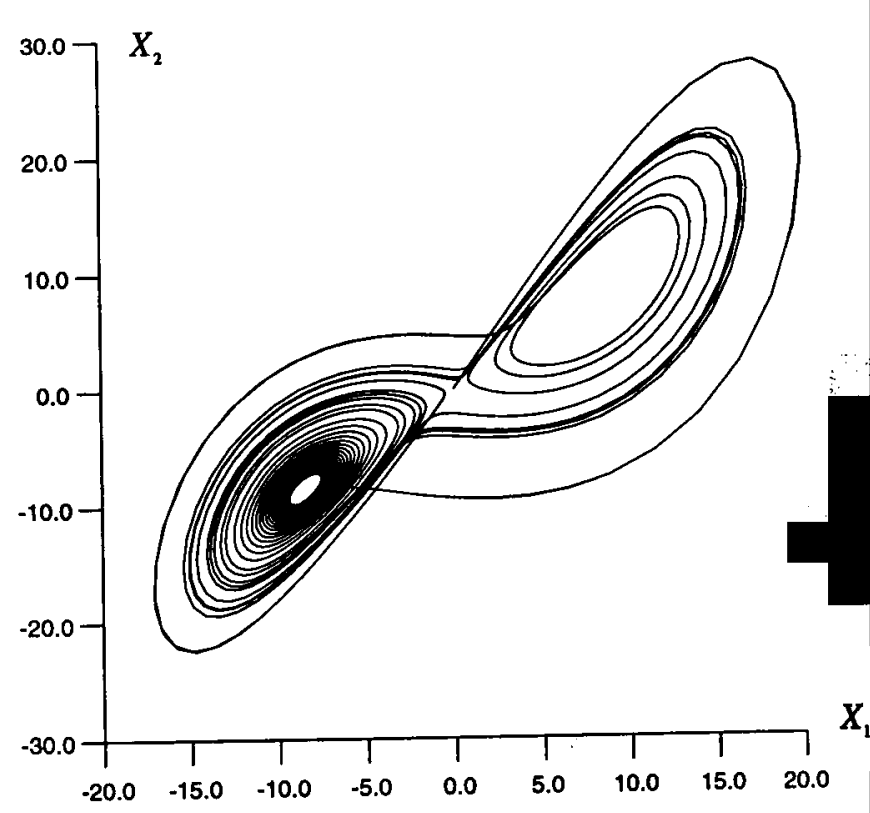

(a)

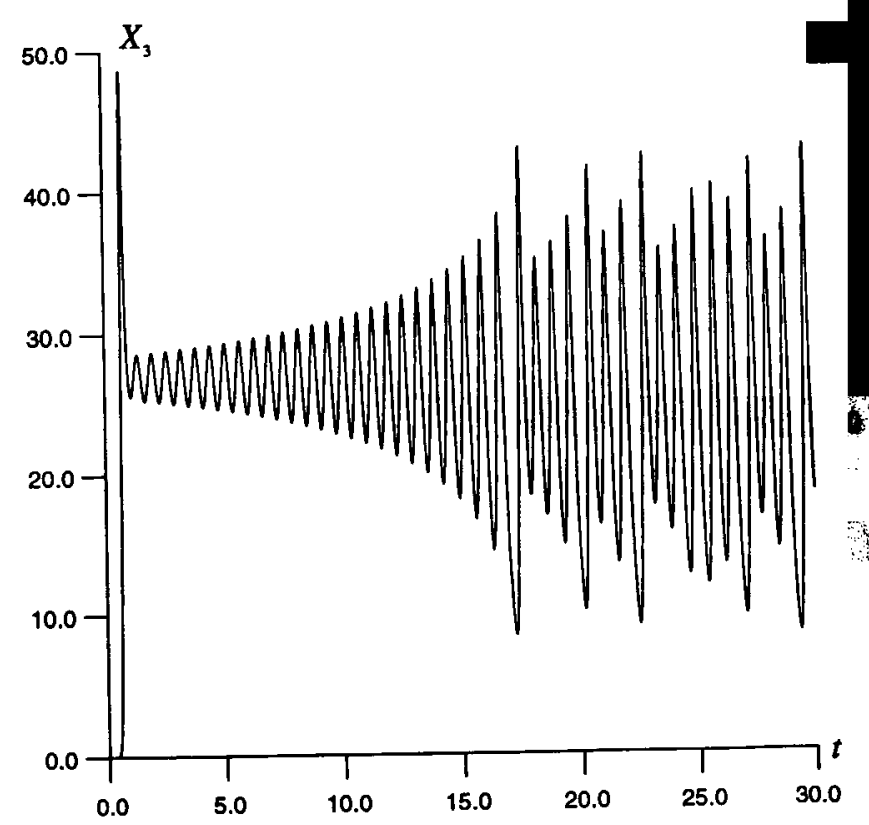

(b)

Fig. 3. Lorenz attractor without white noise: (a) butterfly pattern of track in $\left(X_{1}, X_{2}\right)$ phase space, (b) time series of $X_{3}$. 


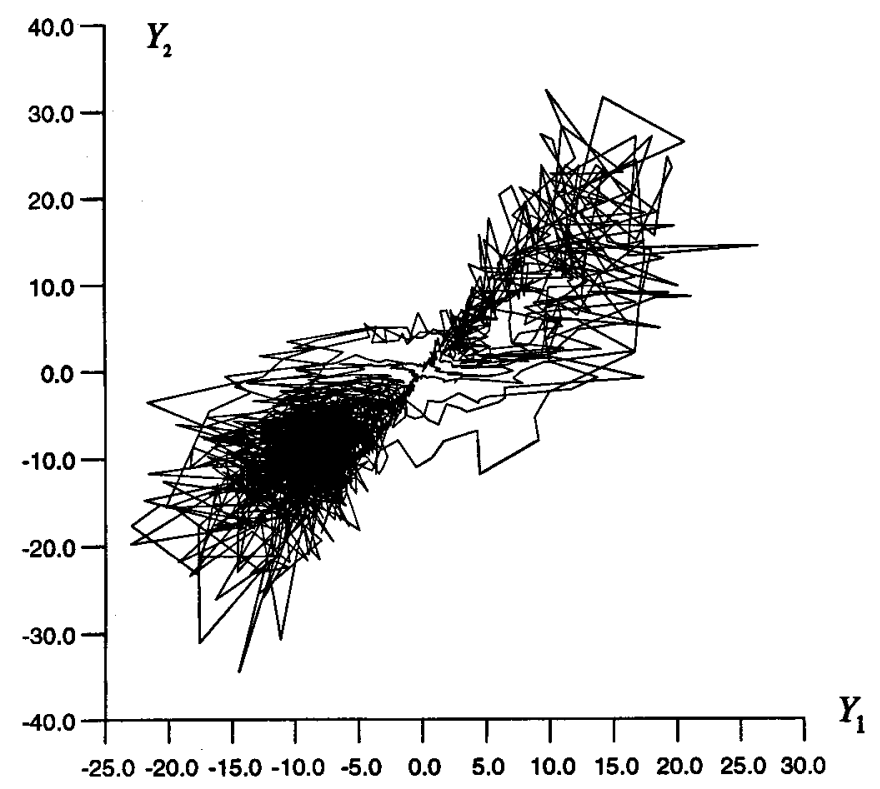

(a)

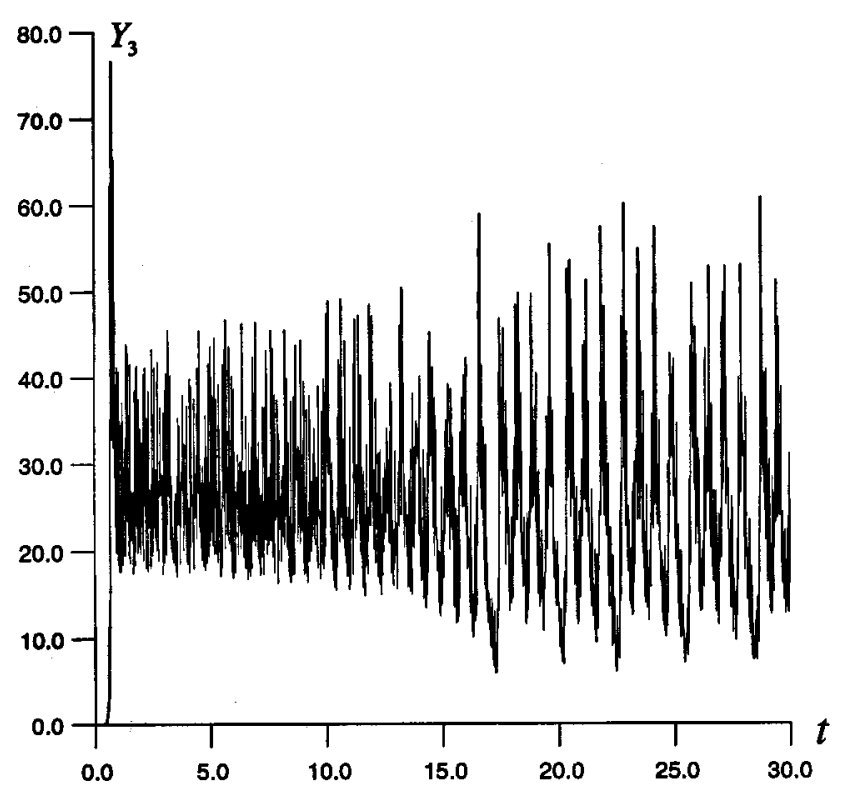

(b)

Fig. 4. Lorenz attractor distorted by white noise with $\eta_{1}=$ 0.6: (a) noisy track in $\left(Y_{1}, Y_{2}\right)$ phase space with no butterfly pattern, (b) time series of $Y_{3}$.

gives the state vector $\mathbf{X}^{(n-1)}(t)$ for the $(n-1)$ th iteration. If the estimated parameter vector $\hat{\mathbf{a}}^{(n-1)}$ has an increment $\Delta \mathbf{a}$, the state vector has a corresponding increment,

$$
\Delta \mathbf{X}^{(n-1)}(t)=\mathbf{U}^{(n-1)} \Delta \mathbf{a}
$$

where $\mathrm{U}$ is the sensibility matrix defined by $U_{i j}=$ $\partial X_{i} / \partial a_{j} ; i, j=1,2,3$. When the increment $\Delta \mathbf{a}$

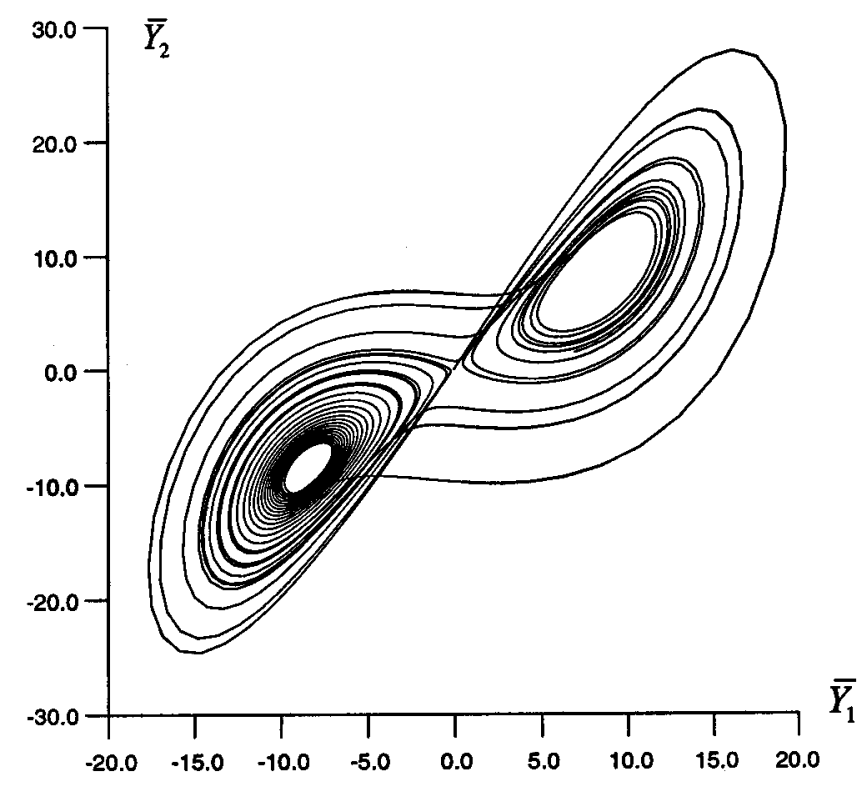

(a)

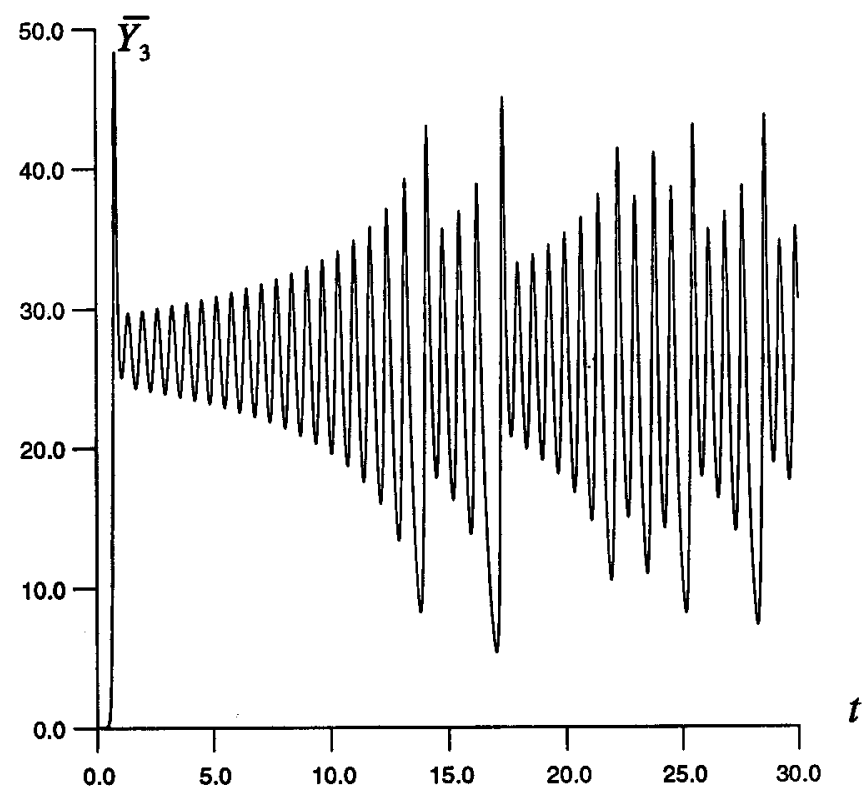

(b)

Fig. 5. Reconstructed Lorenz attractor from the "noisy data" with $\eta_{1}=0.6$ using the rotation method: (a) butterfly pattern of track in $\left(\bar{Y}_{1}, \bar{Y}_{2}\right)$ phase space, (b) time series of $\bar{Y}_{3}$.

is selected such that Eq. (19) is satisfied, this leads to

$$
\begin{aligned}
& \int_{t_{0}}^{T}\left(\mathbf{U}^{t}\right)^{(n-1)} \mathbf{U}^{(n-1)} * \Delta \mathbf{a} d t \\
& \quad=\int_{t_{0}}^{T}\left(\mathbf{U}^{t}\right)^{(n-1)} *\left(\mathbf{Y}-\mathbf{X}^{(n-1)}\right) d t
\end{aligned}
$$

The temporally integrated difference between $\mathbf{Y}(t)$ and $\mathbf{X}^{(n-1)}(t)+\mathbf{U}^{(n-1)} \Delta \mathbf{a}$, reaches the minimum 
[see (18)]. Here, the matrix $\mathbf{U}^{t}$ is the transpose of the matrix $\mathbf{U}$. The estimated parameter vector at the $n$th iteration is taken as

$$
\hat{\mathbf{a}}^{(n)}=\hat{\mathbf{a}}^{(n-1)}+\Delta \mathbf{a} .
$$

We solve Eq. (21) with $\hat{\mathbf{a}}^{(n)}$ and get the state vector at the $n$th iteration, $X(n)(t)$.

At each iteration step, an ill-posed algebraic equation for $\Delta \mathbf{a}$ [Eq. (23)] should be solved. As for the two previous examples, the noise-to-signal ratio and the condition number are large,

$$
\eta_{1} \simeq 0.6, \quad \eta_{3} \simeq 10^{4} \text {. }
$$

The rotation method is used to get minimum $\left(\hat{\eta}_{1}\right.$, $\left.\hat{\eta}_{3}\right)$ at each iteration step. The ill-conditioning of Eq. (23) is greatly reduced because

$$
\hat{\eta}_{3}<5
$$

at all iteration steps.

The iteration converges fast. The final estimated parameter vector is given by

$$
\hat{\mathbf{a}}=\left(\begin{array}{c}
9.93 \\
27.98 \\
2.667
\end{array}\right) \text {. }
$$

After the reconstruction, we obtain the butterfly pattern of the track in the phase space $\left(\mathbf{X}_{1}, \mathbf{X}_{2}\right)$ [Fig. 5(a)] and the unstable oscillation for temporal variation of $X_{3}$ [Fig. 5(b)]. Comparison between Figs. 3 and 5 demonstrates the capability of the rotation method for reconstructing noisy data.

\section{Conclusions}

(1) The rotation method has two major advantages in reconstruction of process and field from imperfect data. First, it does not need any a priori knowledge of noise statistics and/or representation for deterministic perturbations. Second, it reduces the condition number and noise-to-signal ratio drastically, and in turn reduces the imperfection of the system.

(2) The rotation method has capability to effectively reconstruct the linear processes with ranges of $0 \leq \eta_{1} \leq 4-5 ; 0 \leq \eta_{2} \leq 0.5-0.6 ; \eta_{3} \leq 10^{6}$, and the nonlinear Lorenz attractor with ranges of $0 \leq \eta_{1} \leq 0.6 ; 0 \leq \eta_{2} \leq 0.5-0.6 ; \eta_{3} \leq 10^{4}$ at each iteration.

(3) The procedure can be applied to processes and fields with both stochastic and deterministic perturbations. Behavior of the scalar product
$\left(\mathbf{S Q} \overline{\mathbf{Y}}^{*} \mathbf{S Q Y} \mathbf{Y}^{\prime}\right) \rightarrow 0$ determines the effectiveness of noise reduction. Besides, it can be easily generalized on stochastic signals if there is no correlation between $\bar{Y}$ and $Y^{\prime}$.

\section{Acknowledgments}

The primary support of Leonid Ivanov and Tatyana Margolina came from the Ukrainian Academy of Sciences. Leonid Ivanov also thanks the Office of Naval Research (ONR) International Field Office of Europe for support under grant N 00014-99-1-4007. Peter Chu was supported by the Naval Oceanographic Office and the Naval Postgraduate School.

\section{References}

Bennett, A. F. [1992] Inverse Methods in Physical Oceanography (Cambridge University Press, NY) $346 \mathrm{pp}$.

Chu, P. C. [1999] "Two kinds of predictability in Lorenz system," J. Atmos. Sci. 56, 1427-1432.

Eremeev, V. N., Ivanov, L. M., Kirwan, Jr., A. D., Melnichenko, O. V., Kochergin, S. V. \& Stanichnaya, R. R. [1992] "Reconstruction of oceanic flow characteristics from quasi-Lagrangian data, Part 2: Characteristics of the large-scale circulation in the Black Sea," J. Geophys. Res. 97, 9743-9753.

Eykhoff, P. [1973] System Identification: Parameter and State Estimation (Elsevier, Amsterdam), $555 \mathrm{pp}$.

Ivanov, L. M., Kirwan, Jr., A. D. \& Margolina, T. M. [2001] "Filtering noise from oceanographic data with some applications for the Kara and Black Seas," $J$. Mar. Syst. 28, 113-139.

Lorenz, E. N. [1963] "Deterministic nonperiodic flow," J. Atmos. Sci. 20, 130-141.

Strakhov, V. N. [1991] "Method for filtration of the linear algebraic systems as basis for the solutions of linear problem in the gravimetry and magnitometry," Dok. Akad. Nauk SSSR 320, 590-599 (in Russian).

Tarantola, A. [1987] Inverse Problem Theory: Methods for Data Fitting and Model Parameter Estimation (Elsevier), $613 \mathrm{pp}$.

Thiebaux, H. J. \& Pedder, M. A. [1987] Spatial Objective Analysis with Applications in Atmospheric Science (Academic Press, London), 299 pp.

Tikhonov, A. N. \& Arsenin, V. I. [1979] Methods for Solving Ill-posed Problems (Nauka, Moscow), 285 pp.

Tikhonov, A. N., Goncharsky A. V., Stepanov V. V. \& Yagola, A. G. [1990] Numerical Methods for Ill-posed Problems (Nauka, Moscow), 229 pp. 